\title{
Switching Direction of Liquid Flow by Amplification of Wetting Properties of Partilly Roughened Prism Array
}

\author{
Hyemin Lee ${ }^{1} \&$ Hyunsik Yoon ${ }^{1}$ \\ ${ }^{1}$ Department of Chemical and Biomolecular Engineering, Seoul National University of Science \& Technology, \\ Seoul, 139-743, Korea \\ Correspondence: Hyunsik Yoon, Department of Chemical and Biomolecular Engineering, Seoul National \\ University of Science \& Technology, Seoul, 139-743, Korea. E-mail: hsyoon@seoultech.ac.kr
}

Received: June 26, 2015 Accepted: July 28, 2015 Online Published: August 20, 2015

doi:10.5539/jmsr.v4n4p43 URL: http://dx.doi.org/10.5539/jmsr.v4n4p43

\begin{abstract}
Superhydrophilic and superhydrophobic surfaces inspired from nature have been developed for anti-fogging, anti-reflecting, water-repelling, anti-icing and self-cleaning films. The amplification of the hydrophilicity and hydrophobicity originates from surface roughness, particularly from their multiscale geometry. In this paper, we present a strategy to exploit the enhancement of wetting properties on roughened surfaces for switching the direction of unidirectional liquid flow on an opened microchannel. We exploit a unidirectional liquid spreading on a hierarchical two-face prism array, which have the symmetry in microscale while having the asymmetry in nanoscale. Based on the switching the direction of the lower critical angle, the transition of the surface chemistry results in the change of flow directions in an open channel.
\end{abstract}

Keywords: prism, superhydrophobicity, superhydrophilicity, directional flow, polymer, contact angle

\section{Introduction}

Superhydrophilic and superhydrophobic surfaces can be observed in daily life and nature such as a paper, sponge, plant leaf, leg of water strider. These surfaces have been developed for functional application including anti-fogging, anti-reflection or anti-sticking, self-cleaning and anti-icing films (Barthlott et al., 1997; Blossey et al., 2003; Choi et al., 2011; Feng et al., 2005; Quéré et al., 2008; Verplanck et al., 2007; Xia et al., 2008). It is well known by Wenzel and Cassie-Baxter models (Quéré et al., 2008) that the wetting properties such as hydrophilicity and hydrophobicity can be amplified by increasing surface roughness. Especially, hierarchical structures inspired from lotus leaves have been developed for their superhydrophobicity and water repellency (Barthlott et al., 1997; Blossey et al., 2003). Although the surface with superhydrophilicity or superhydrophobicity can be used as a special coating by themselves, there still remains needs for new and emerging applications such as microfluidic devices, cell arrays, and separation of oil and water (Ueda et al., 2013). Here, we propose a strategy exploiting a hierarchical structure with the symmetry in microscale while having the asymmetry in nanoscale to manupulate liquid flow direction on an open channel. By using the mechanism of the critical contact angle on an edge (Kim et al., 2013; Oliver et al., 1977; Yoon et al., 2015), we could switch the flow direction due to the change in the direction of lower contact angle. Since the contact angle difference originates from the difference of roughness between adjoining surfaces, the strategy has an advantage of the wide range of material choices for the manipulation of liquid flow.

\section{Methods}

\subsection{Materials}

We used a homogeneous UV-curable liquid prepolymer by mixing multi-functional acrylated prepolymer (Ethoxylated Trimethylolpropane triacrylate, M3130, Miwon Specialty Chemical Co., Ltd.) with acrylated-functionalized polysiloxane (Ac-PSi) (Silicon polyether acrylate, Rad2200N, Degussa). As a photoinitiator, we added Darocur 1173 (2-hydro-2-methyl-1-phenyl-1-propane, Ciba Specialty Chemicals, Switzerland). The detail blend ratio could be found elsewhere (Choi et al., 2011).

\subsection{Polymeric Prism Array}

We prepared a nickel prism master by conventional mechanical machining. After preparing a master, liquid prepolymer mixture of acrylated prepolymer and acrylated-functionalized polysiloxane (Ac-PSi) was dropped 
onto a master and a transparent PET film was placed on the prepolymer. The UV-curable prepolymer mixture was crosslinked by ultra-violet (UV) light exposure. Then, the crosslinked polymeric prism array was detached from the master.

\subsection{Two Face Prism Array}

The polymeric prism array ( 20 or $50 \mu \mathrm{m}$ in period, $45^{\circ}$ in the prism angle) was deposited with $20 \mathrm{~nm}$ thick gold film only on one face of the prism array by oblique metal deposition. Gold films deposited by a conventional thermal evaporator and we use inclined loader to get an oblique angle of around $50^{\circ}$. During the evaporation, metal atoms were guided vertically, with the inclined loader defining the oblique angle. The detail experimental procedure can be found elsewhere (Kim et al., 2013; Yoon et al., 2009, 2015).

\subsection{Two Face Prism Array}

The prepared two-face prism array was roughened by oxygen plasma. The gold-deposited faces are not etched by oxygen plasma because the metal films behaves as an etch mask. On the other hand, polymeric face has nanoroughness because of selective etching of polymer while the silicon moiety acted as another etch barrier. The detail experimental data could be found elsewhere (Yoon et al., 2015).

\subsection{Surface Treatments}

To make surface hydrophobic, the multiscale two-face prism arrays were treated with a liquid phase self-assembled monolayer (SAM) species of fluoroalkylsilane under an ambient atmosphere. The patterned substrate was dipped for several minutes into tridecafluoro-1,1,2,3-tetrahydrooctyl-1-triethoxysilane that was mixed with perfluorocarbon fluid, FC-40 (3M Co., Ltd, USA) as a diluent. Then, the substrate was placed in an oven at $120^{\circ} \mathrm{C}$ for $10 \mathrm{~min}$ to remove excess diluent completely as well as to ensure bonding of $-\mathrm{OH}$ functional groups on oxidized surfaces.

\subsection{Measurements}

To measure wetting properties, advancing and receding contact angles (CAs) of water droplets were measured at ambient atmosphere using a contact angle analyzer (DSA100, Kruss GmbH., Germany) by the sessile drop method with increasing or decreasing droplet volume. The CA values presented in this experiment were averaged over five different positions.

\section{Results and Discussion}

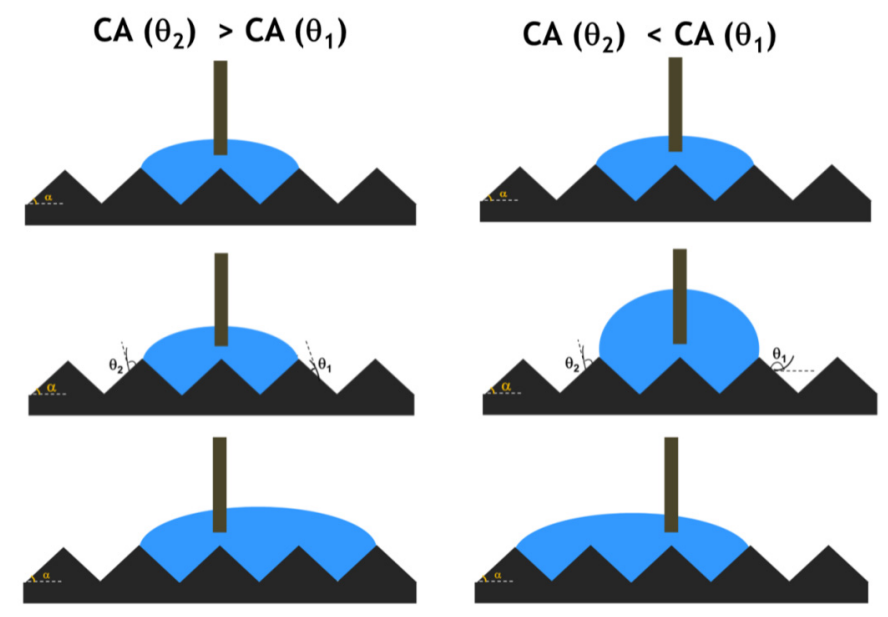

Figure 1. Schematic illustration for switching the flow direction in a prism array

Figure 1 is a schematic illustration of the strategy of switching the flow direction by the concept of critical contact angles with the three-phase contact line across the ridges of channels created by the prism array. As we discussed in our previous works (Kim et al., 2013, Yoon et al., 2015), the liquid-front is pinned on prism edges and the pinning is continued during increasing liquid volume until the contact angle of the liquid exceeds its critical contact angle on the edge. The critical contact angle $\theta_{c}$ can be derived as follows (Kim et al., 2013, Oliver, et al., 1977; Yoon et al., 2015): 


$$
\theta_{\mathrm{c}}=\theta_{*}+\alpha
$$

where $\theta^{*}$ is the contact angle on a planar surface and $\alpha$ is the prism angle. In the hierarchical two-face prism system, the contact angles of the two-faces are different because of their different morphologies. As a result, when the contact angle on the right face $\left(\theta_{1}\right)$ is lower than the angle on the left face $\left(\theta_{2}\right)$, the liquid flows to the right direction because the critical angle is lower in the right face. When we change the contact angle $\left(\theta_{1}\right)$ of the right face to be greater than $\theta_{2}$ by proper chemical treatments, the direction of lower critical angle is switched to the left face. The novelty of the hierarchical two-face prism arrays is that the capability of switching liquid flow direction by exploiting the transition of the surfaces from hydrophobic to hydrophilic to switch the direction of lower critical contact angle.

(a)

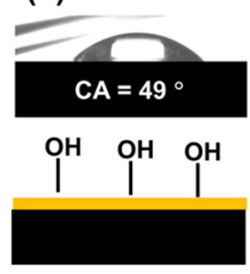

(e)

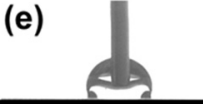

$\mathrm{CA}_{\text {Critical }}=110$ (b)
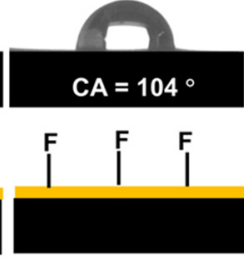

(c)
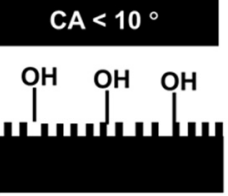

(d)

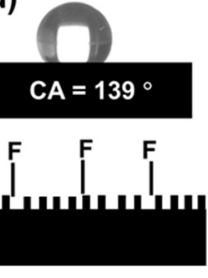

(g)

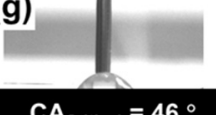

(h)

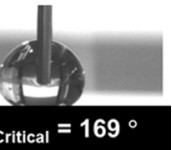

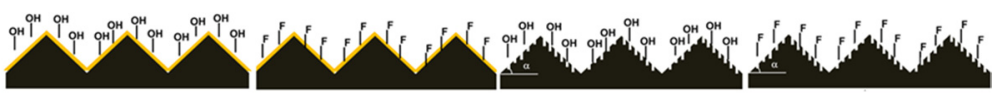

Figure 2. Pictures representing contact angles on planar surfaces (a-d) and critical angles (e-h)

As shown in Figure 2(a) and 2(b), the contact angle on a planar gold surface after oxygen plasma is $\sim 50^{\circ}$ and changed to $\sim 100^{\circ}$ after a fluorinated self-assembled monolayer (SAM) treatment. The prism surface then becomes hydrophilic again by treating it with oxygen plasma at the mild condition ( $25 \mathrm{~W}$ for $10 \mathrm{sec})$. We also noted that the hydrophilic transformation by oxygen plasma treatment involves the ozone cleavage of C-F bonds and the substitution of $-\mathrm{OH}$ groups or functionalization to $-\mathrm{OH}$ groups. As shown in Figure 2(c) and 2(d), the contact angles on roughened surfaces are significantly changed from $5^{\circ}$ to $139^{\circ}$ by switching the surface chemistry from hydrophilic to hydrophobic. The amplification of hydrophobicity and hydrophilicity could be explained by Wenzel's model (Quéré et al., 2008). Figure 2(e-h) shows pictures representing the critical contact angles on symmetric prism arrays coated by $\mathrm{Au}$ film and roughened by the oxygen plasma. The average of the differences between critical contact angles (Figure 2(e-h)) and contact angles (Figure 2(a-d)) is $43.25^{\circ}$, which is in good agreement with the prism angle $\left(45^{\circ}\right)$ derived from Eq. (1).

When the two faces assembled together as a form of the multiscale two face prism array, we can exploit the changes of the direction of lower critical contact angle to switch the flow directions. Figure 3 shows the graph of contact angles on planar surfaces switched by chemical treatments. It is notable that repeating the cycles of SAM/oxygen plasma treatments for more than 5 times shows excellent reversibility in contact angle. As shown in Figure 3, the lower contact angle after hydrophobic treatment is in the smooth side of the Au-coated surface because the contact angle on the roughened surface is greater than that on the smooth surface. On the other hand, the lower contact angle after hydrophilic treatment is in the side of the roughened surface because it is more hydrophilic

From the change of the direction of lower contact angle by transition of the surface from hydrophilic to hydrophobic or vice versa, we expect that the liquid flow direction can be switched. Previously, we reported a scheme of two-face prism array based on stimuli-responsive polymers to switch the flow direction (Kim et al., 2013). Since the range of the contact angles of the polymer should be restricted $\left(45^{\circ} \sim 72^{\circ}\right)$, however, it is difficult to choose a compatible metal film with a contact angle within the range. In the case of the multiscale two-face prism array, it has adjoining smooth and rough surfaces; the contact angle on the rough surface is lower when the entire surface becomes hydrophilic. On the other hand, the contact angle on the smooth surface becomes lower when it is changed to a hydrophobic surface. By exploiting the aforementioned wetting characteristics, the change in flow direction could be achieved with the wide ranges of material choices. 


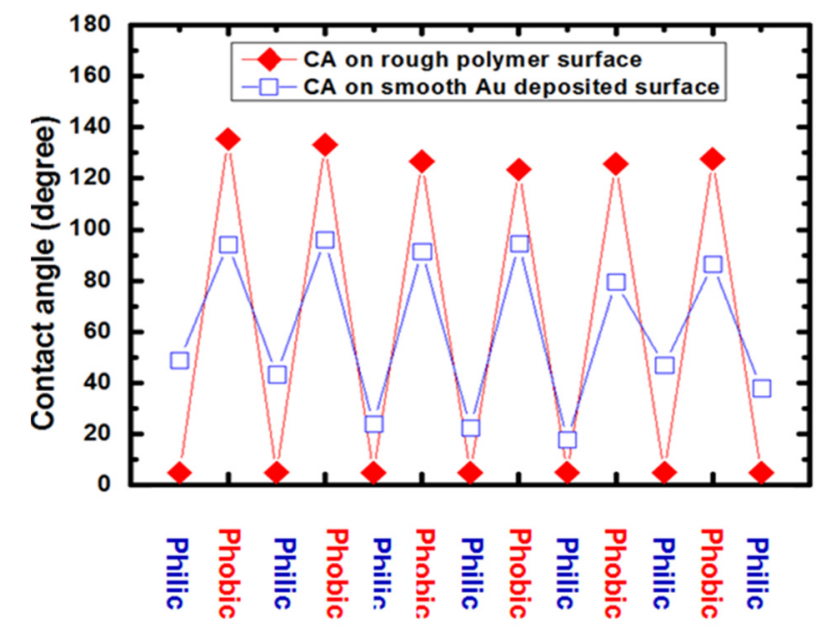

Figure 3. A graph showing reversible switching of contact angles on planar surfaces

(a)
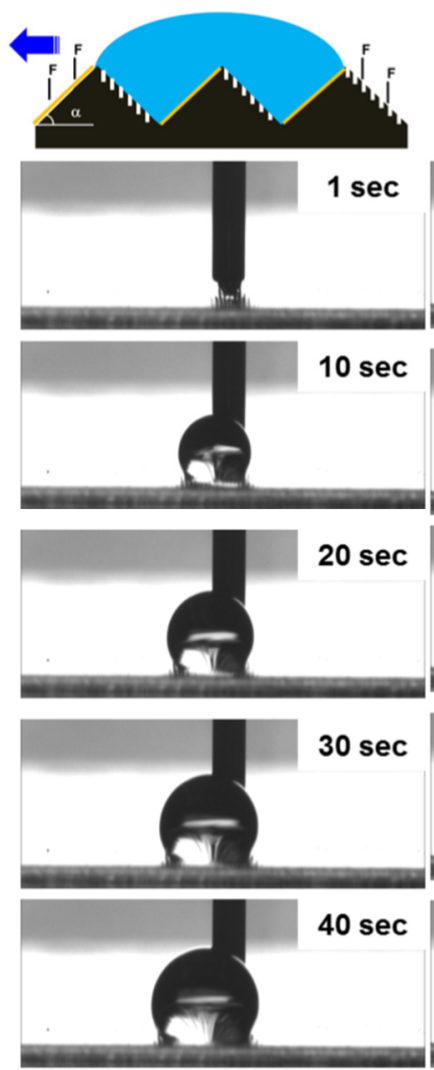

(b)
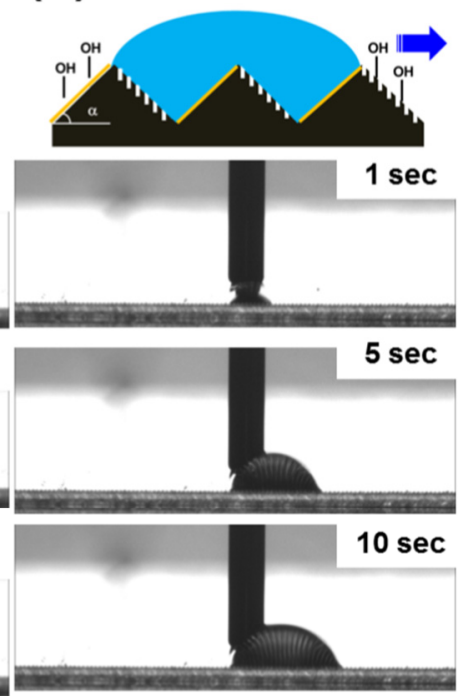

$15 \mathrm{sec}$
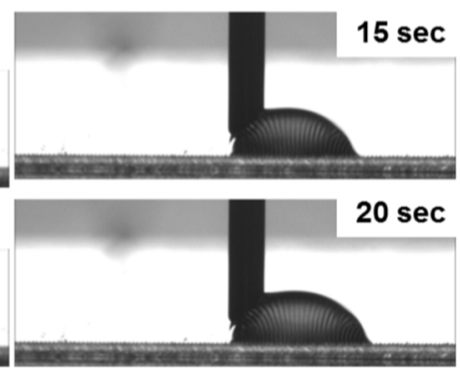

Figure 4. Movie cuts of liquid flow (a) after fluorinated SAM treatment and (b) oxygen plasma treatment

Figure 4 shows the movie cuts for the change in the direction of liquid flow on hydrophobic and hydrophilic two-face prism arrays, respectively, with the same micro- and nano-structures maintained. As shown in Figure 4(a), the liquid flows towards the smooth metal face with a relatively slow speed at a feeding rate of $5 \mu \mathrm{l} / \mathrm{min}$ because of its high critical contact angle in the moving direction. Remarkably, the moving direction was reversed towards the roughened polymer face after the oxygen plasma, as shown in Figure 4(b). Due to the hydrophilic nature of the surfaces, the liquid flow speed was increased at the same feeding rate. Such a reversal of flow direction was repeated more than five times, in good agreement with the similar wetting behavior. 


\section{Conclusions}

In summary, we propose a scheme to switch a flow direction by hierarchical two-face prism arrays which are symmetric in microscale but asymmetric in nanoscale. The prism arrays were used to switch the direction of water flow in reversible manner without deterioration of the hierarchical structures. It was observed that the liquid flows to the direction of the lower critical contact angle. By exploiting the transition of the roughened surfaces from hydrophobicity to hydrophilicity, we further demonstrated the switching of flow direction on pre-defined prism arrays, which would enable the control of the direction of liquid flow on demand.

\section{Acknowledgments}

This study was supported by Seoul National University of Science and Technology.

\section{References}

Barthlott, W., \& Neinhuis, C. (1997). Purity of the sacred lotus, or escape from contamination in biological surfaces. Planta, 202(1), 1-8. http://dx.doi.org/10.1007/s004250050096

Blossey, R. (2003). Self-cleaning surfaces—virtual realities. Nature materials, 2(5), 301-306. http://dx.doi.org/ $10.1038 /$ nmat 856

Choi, S. J., Choi, M. K., Tahk, D., \& Yoon, H. (2011). Fabrication of a hierarchical structure by oxygen plasma etching of a photocured microstructure containing a silicon moiety. Journal of Materials Chemistry, 21(38), 14936-14940. http://dx.doi.org/10.1039/c1jm12305j

Feng, X., Zhai, J., \& Jiang, L. (2005). The fabrication and switchable superhydrophobicity of TiO2 nanorod films. Angewandte Chemie International Edition, 44(32), 5115-5118. http://dx.doi.org/10.1002/anie.2005 01337

Kim, S. M., Koh, J. H., Suh, H. S., Yoon, H., Suh, K. Y., \& Char, K. (2013). Thermoresponsive switching of liquid flow direction on a two-face prism array. Soft Matter, 9(16), 4145-4149. http://dx.doi.org/10.1039/ c3sm27901d

Oliver, J. F., Huh, C., \& Mason, S. G. (1977). Resistance to spreading of liquids by sharp edges. Journal of Colloid and Interface Science, 59(3), 568-581. http://dx.doi.org/10.1016/0021-9797(77)90052-2

Quéré, D. (2008). Wetting and roughness. Annu. Rev. Mater. Res., 38, 71-99. http://dx.doi.org/10.1146/annurev. matsci.38.060407.132434

Ueda, E., \& Levkin, P. A. (2013). Emerging Applications of Superhydrophilic - Superhydrophobic Micropatterns. Advanced Materials, 25(9), 1234-1247. http://dx.doi.org/10.1002/adma.201204120

Verplanck, N., Coffinier, Y., Thomy, V., \& Boukherroub, R. (2007). Wettability switching techniques on superhydrophobic surfaces. Nanoscale Research Letters, 2(12), 577-596. http://dx.doi.org/10.1007/s11671007-9102-4

Xia, F., \& Jiang, L. (2008). Bio-inspired, smart, multiscale interfacial materials. Adv. Mater, 20(15), 2842-2858. http://dx.doi.org/10.1002/adma.200800836

Yoon, H., Jeong, H. E., Kim, T. I., Kang, T. J., Tahk, D., Char, K., \& Suh, K. Y. (2009). Adhesion hysteresis of Janus nanopillars fabricated by nanomolding and oblique metal deposition. Nano Today, 4(5), 385-392. http://dx.doi.org/10.1016/j.nantod.2009.08.007

Yoon, H., Sung, S. H., Koh, J. H., Kim, S. M., Choi, S. J., Suh, K. Y., \& Char, K. (2015). Directional step flow across ridges on multiscale two-face prism array. Macromolecular Research, 23(2), 145-148. http://dx.doi.org/10.1007/s13233-015-3026-0

\section{Copyrights}

Copyright for this article is retained by the author(s), with first publication rights granted to the journal.

This is an open-access article distributed under the terms and conditions of the Creative Commons Attribution license (http://creativecommons.org/licenses/by/3.0/). 